\title{
Multi-Impairment WDM Optical Performance Monitoring for Burst Switched Networks.
}

\author{
Lamia Baker-Meflah Student Member, IEEE, Benn C. Thomsen, John E. Mitchell, Member, IEEE, \\ Polina Bayvel, Fellow IEEE
}

\begin{abstract}
We present an analytical investigation of a performance monitoring scheme capable of simultaneously monitoring Chromatic Dispersion, Polarization Mode Dispersion and Optical Signal to Noise Ratio at 40Gbit/s on timescales that are appropriate to optical burst switched networks. This analysis supports previously published experimental results which showed that this technique is scalable to WDM network monitoring.
\end{abstract}

Index Terms - Optical Performance Monitoring, Optical Burst Switching

\section{INTRODUCTION}

$\mathrm{A}^{\mathrm{s}}$ s higher bit-rates and WDM optical transmission technologies have evolved, the network bottleneck has moved from the optical-transport to the routing layer, as conventional electronic routers have not been capable of offering a cost-effective solution to the increasing bandwidth demand [1]. To cope with this, highly flexible and dynamic networks were envisioned a few years ago to provide virtually unlimited bandwidth with dynamic reconfiguration supported not only in the electrical layer, but also in the optical layer. Current research has shown that dynamically reconfigurable networks, with an optical plane consisting of optical transmission and optical switching capabilities, can yield to a more efficient utilization of network resources, however, at the cost of increased complexity of required components and routing $[2,3]$. These networks require a number of novel technologies to ensure their dynamic reconfigurability. One of the technologies required is optical performance monitoring (OPM) to address both fault management and to control transmission. New forms of physical layer functionalities, such as real-time impairment monitoring and compensation (i.e. monitoring and compensation performed on the network switching timescales) and dynamic reconfiguration, introduce greater complexity that may demand sophisticated OPM to monitor the quality of the optical signal throughout the network. OPM can therefore be seen as crucial to the success of next generation optical networks [4]. When considering impairments due to chromatic dispersion in static networks, it

Manuscript received April 14, 201-. This work was supported in part by the EU FP6 Project NOBEL and the UK Engineering and Physical Sciences Research Council (EPSRC). L. Baker-Meflah B. C. Thomsen, J. E. Mitchell and $\mathrm{P}$ Bayvel, are with the Department of Electronic and Electrical Engineering, University College London, Torrington Place, London, WC1E 7JE (e-mail:j.mitchell@ee.ucl.ac.uk).

Copyright (c) 2009 IEEE. Personal use of this material is permitted. However, permission to use this material for any other purposes must be obtained from the IEEE by sending a request to pubs-permissions@ieee.org. is sufficient to monitor a single channel in a WDM system as all channels travel through the same fixed path accumulating the same residual chromatic dispersion. However, in dynamic networks, each channel will traverse a unique path through the network thus the channels arriving at the monitoring point will, in general, exhibit different amounts of residual dispersion. Therefore, it is necessary to monitor all channels individually to quantify the degradation, without the requirement of knowing the data path history. The monitoring feature can then be used in conjunction with a dispersion compensation device which can either be optical, or electrical, or used to trigger real-time alarms for traffic re-routing. Figure 1 depicts a dynamically reconfigurable network schematic using OPM where dispersion compensation fiber (DCF) is typically incorporated in each link of the network to compensate for the chromatic dispersion due to single-mode fiber (SMF), leaving the signal subject to some residual dispersion. In this scheme, the OPM device is also deployed in each link and is connected to a tunable dispersion compensator (TDC). The combination of the OPM and TDC is used to monitor and compensate for residual dispersion. It can also be envisaged that the OPM device could be an integral part of the network management system, allowing for signal rerouting when monitoring a signal failure. The system manufacturers would then design and integrate the OPM function into the optical network sub-systems that would be deployed by network operators.

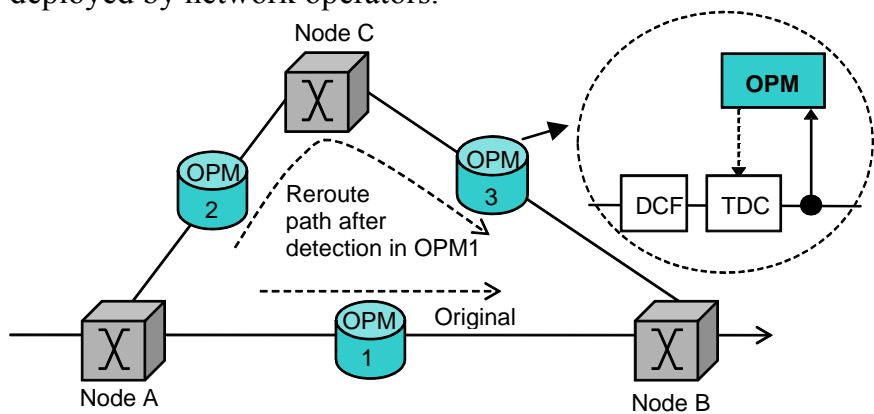

Fig 1: General schematic of a dynamically reconfigurable network, depicting the possible location of optical performance monitoring (OPM) and tunable dispersion compensation (TDC) devices. The rerouted path is performed after failure detection in OPM1.

For OPM to be an enabling technology of dynamic networks a number of criteria should be met: Maximum coverage of impairments: Various impairments should be able to be monitored simultaneously and independently including OSNR, CD, PMD and power. WDM: Multiple channels should be simultaneously monitored with the minimum 
duplication of optical and electrical components, as this will reduce the cost per channel. Range and accuracy: Dynamic reconfiguration puts new constraints on the OPM measurement range and accuracy which are dictated by the network architecture. Response time: In a dynamic network, the OPM measurement time must be of the same order of magnitude as the network's switching time which becomes faster when moving toward packet architectures. Nonintrusive: Must be implemented so that the optical signal is not terminated (a tap used). The monitor's power sensitivity is important in limiting the penalties introduced by the OPM into the system. Network agnostic: Bit-rate and modulation format independent and does not require transmitter modification.

In this paper we present a technique that meets all of these requirements, simultaneous monitoring $\mathrm{PMD}, \mathrm{CD}$ and OSNR at nanosecond speeds without modification to the transmitter that can be scaled to multiple WDM channels without replication of any high-speed component. Although demonstrated for $40 \mathrm{Gbit} / \mathrm{s}$ NRZ systems it can be easily adapted for other rates and modulation schemes. This analysis supports previously published experimental results [5]. A wide variety of optical performance monitoring methods have been proposed and a comprehensive review is available in [6] Section II describes the monitoring system proposed. Section III provides an analytical derivation of the function of the system and demonstrated that CD, PMD and OSNR can be monitored independently. Section IV provides an experimental demonstration of the technique and Section $\mathrm{V}$ gives the conclusions of this work.

\section{SYSTEM CONCEPT}

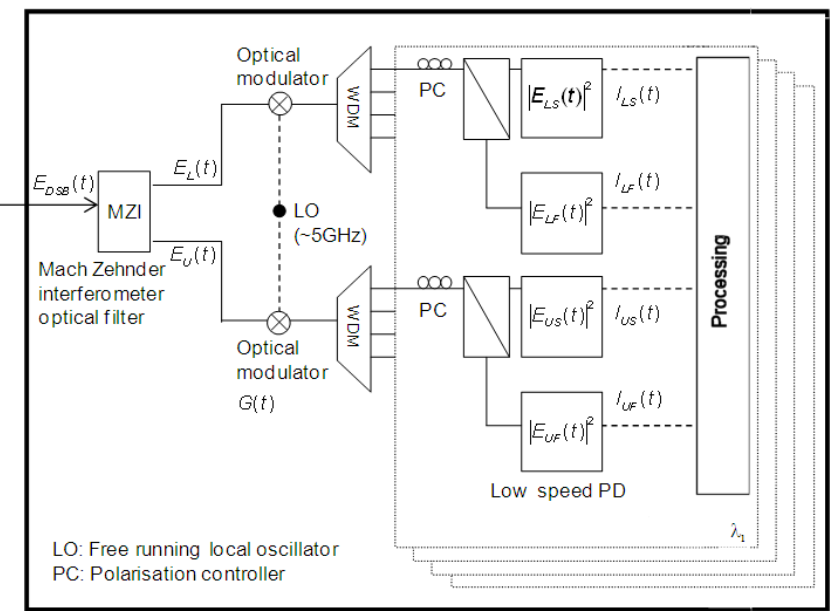

Fig 2: Implementation of the proposed OPM technique used for simultaneous $\mathrm{CD}, \mathrm{PMD}$ and OSNR monitoring.

The multichannel OPM system is illustrated in figure 2 and the measurement principle for 1 st order chromatic dispersion (CD), polarization mode dispersion (PMD) and optical signalto-noise ratio (OSNR) is shown schematically in Figure 3 for a single channel. The measurement is based on determining the relative phases of a selected RF tone that is present in the signal due to the data modulation for PMD and CD measurements; and the tones' amplitude relative to the signal average-power for OSNR measurements. The signal is optically filtered using a partial bit-delay Mach-Zehnder interferometer (MZI) to select the optical carrier and a single sideband. The free spectral range (FSR) of the MZI is chosen to be twice the frequency spacing between the upper and lower sidebands and tuned so that the filter transmits one sideband on its first output and the other sideband on the its second output. Under these conditions the upper and lower sidebands signals are obtained simultaneously from the two outputs of the MZI. For multi-wavelength operation it is also necessary to ensure that the FSR is a sub-multiple of the WDM channel spacing, such that the first output contains the upper sideband signals of each WDM channel and similarly the second output contains the lower sideband signals. Each single sideband (SSB) signal is then electro-optically heterodyned down to an intermediate-frequency (IF) in the order of a few $\mathrm{kHz}$ (compared to the monitored tone of a few $\mathrm{GHz}$ ). The use of electro-optically heterodyning enables the simultaneous down conversion of all WDM channels in a single device and thus limits the required number of high bandwidth devices. A polarizing beam splitter (PBS) is used to separate each SSB signal into two orthogonal polarization states, which are then detected using four low bandwidth square-law photodetectors (note only one sideband is depicted in Figure 3 but both sidebands are processed in the same way). For multichannel WDM operation the signals, from the outputs of the EAMs are first wavelength demultiplexed before the polarizations are separated. The detected electrical signals are then digitized and the IF tones are extracted in software with a fast Fourier transform (FFT).

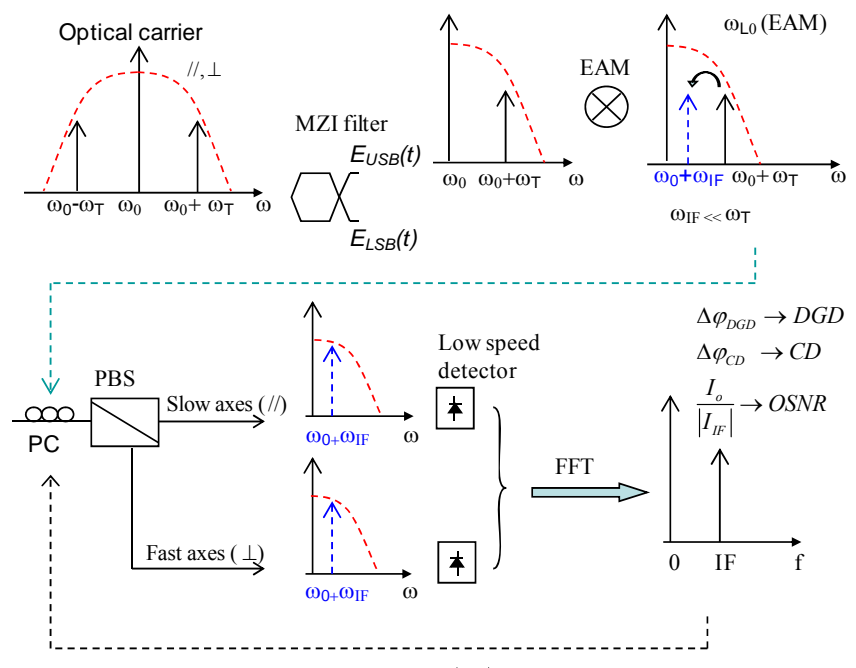

Feedback signal: IF tone RF power $\left|I_{I F}\right|$

Fig 3: Principle of the spectral analysis method after frequency downconversion (only the USB sideband is depicted for detection). The dotted tone indicates the down-converted tone at the IF frequency. $\omega_{0}$ represents the optical carrier frequency, $\omega_{T}, \omega_{I F}$ and $\omega_{L O}$ represent respectively the frequency of the monitored tone, the intermediate frequency and the local oscillator used to drive the optical modulators

\section{ANALYTICAL MODEL}

Here an analytical expression for the three impairments; CD, PMD and OSNR is derived. We describe the optical signal 
and noise, using a Fourier series representation, as a finite number of tones located within the signal optical bandwidth that are separated by a constant frequency spacing $\Delta \omega$. The spacing between the tones depends on the length of the repeating pseudo-random bit-sequence (PRBS) data signal. In the case of a completely random data stream, $\Delta \omega$ tends to zero and the data tones become a continuum. The envelope of the electric field $E(t)$ of a binary optical signal may be described in the time domain as [7]:

$$
E(t)=\left(\sum_{k=-\infty}^{\infty} b_{k}(p(t) \otimes \delta(t-k T))\right) \exp \left(i\left(\omega_{0} t+\varphi_{0}\right)\right)
$$

where $b_{k}=0,+1$ is the transmitted random data stream, $p(t)$ is the pulse shape, $T$ is the signal's bit period and $\delta$ represents the Dirac function. The analysis simplifies considerably with the use a Fourier series representation for field. Thus $E(t)$ is expanded in terms of Fourier series coefficients $\alpha_{k}$, with the amplified spontaneous emission noise represented in the same way [8]. We band-limit the signal to twice the signal bit-rate $2 / T$ using an ideal band-pass filter with a unity transfer function inside the band-pass and zero outside it (the phase effect of the filter is neglected). The dual-sideband electric field $E_{D S B}(t)$ is then given by:

$$
E_{D S B}(t)=\sum_{k=-M}^{M} \alpha_{k} \cos \left(\omega_{k} t+\varphi_{k}\right)+\sum_{k=-M}^{M} n_{k} \cos \left(\omega_{k} t+\varphi_{r k}\right)
$$

where $\alpha_{k}, \omega_{k}$ and $\varphi_{k}$ are, respectively, the amplitude, the optical angular-frequency and the phase of the signal's $k^{\text {th }}$ tone; $n_{k}$ and $\varphi_{r k}$ are, respectively, the amplitude and the random phase of the noise's $k^{\text {th }}$ tone and $M$ is defined such that $2 M+1$ is the total number of tones present within the considered bandwidth of the signal.

In the presence of first order PMD, the modulated dualsideband signal $E_{D S B}(t)$ can be modeled within the signal's bandwidth as the sum of monochromatic tones which are all linearly polarized light. Nonlinear transmission effects are neglected here. Without loss of generality, it is assumed that the fast and slow principal states of polarizations (PSP) [9] can be represented by a column vectors $\left[\begin{array}{l}1 \\ 0\end{array}\right]$ and $\left[\begin{array}{l}0 \\ 1\end{array}\right]$ respectively, such that a linearly polarized signal can be represented as (4) where $\gamma=\sin ^{2} \phi$ where $\phi$ represents the angle between the linearly polarized signal and the fast principal state; $\gamma$ represents the $1^{\text {st }}$ order PMD power-splitting-ratio; $\Delta \tau$ is the DGD, defined as the group-delay difference between the slow and fast modes. It is noted that the phase on both PSPs for each tone within the signal bandwidth are equal since the signal is considered to be linearly polarized. The noise power is split equally between both PSP.

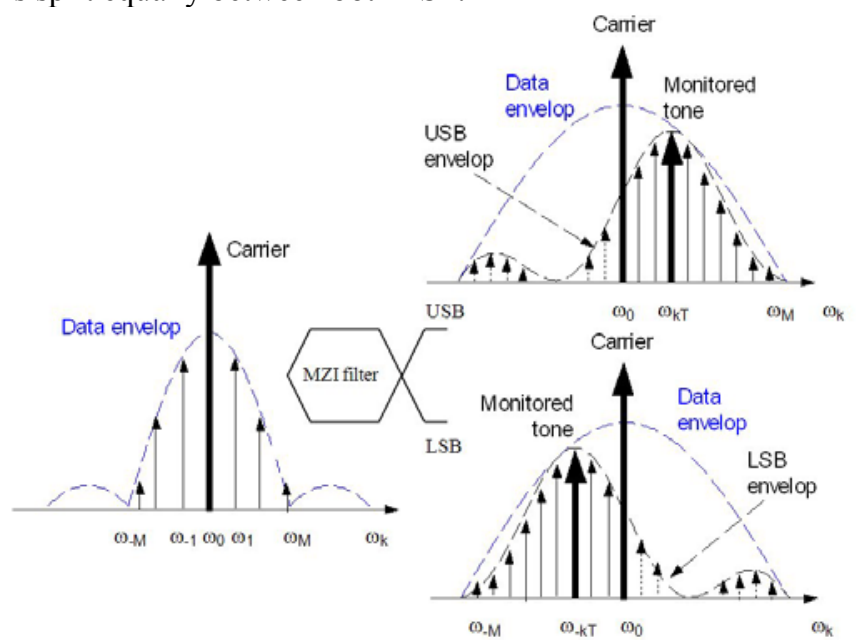

Figure 4: Power spectrum diagram of a NRZ DSB input signal centred around the carrier optical frequency $\omega 0$, LSB and USB output signals after an MZI filter ( $\mathrm{k}$ is the tone)

\section{A. Monitoring function.}

A MZI is used to filter the DSB signal into lower-sideband (LSB) and upper-sideband (USB) signals as demonstrated by the power spectra shown in Figure 4. In deriving the model, we assume an ideal sideband filter and thus the only tones that are considered are from $-\mathrm{M}$ to 0 for the LSB and from 0 to $\mathrm{M}$ for the USB where $k_{T}$ is the monitored tone index. The expression of each sideband is given by (3) and (4) where $T_{k}$ is the filter's amplitude at the $k^{\text {th }}$ tone. In a WDM system, all channels are simultaneously optically down-converted using two electro-absorption modulators (EAM) (one for each sideband). The two polarization components of each of the down-converted signals are then extracted using a PBS which is preceded by a polarization-controller as shown in Figure 4.

$$
\begin{gathered}
\overrightarrow{E_{D S B}}(t)=\left(\begin{array}{l}
\cos \phi \sum_{k=-M}^{M} \alpha_{k} \cos \left(\omega_{k}\left(t-\frac{\Delta \tau}{2}\right)+\varphi_{k}\right) \\
\sin \phi \sqrt{\gamma} \sum_{k=-M}^{M} \alpha_{k} \cos \left(\omega_{k}\left(t+\frac{\Delta \tau}{2}\right)+\varphi_{k}\right)
\end{array}\right)+\left(\begin{array}{l}
\frac{1}{\sqrt{2}} \sum_{k=-M}^{M} n_{k} \cos \left(\omega_{k} t+\varphi_{x r k}\right) \\
\frac{1}{\sqrt{2}} \sum_{k=-M}^{M} n_{k} \cos \left(\omega_{k} t+\varphi_{y r k}\right)
\end{array}\right) \\
\overrightarrow{E_{L S B}}(t)=\left(\begin{array}{l}
\cos \phi \sum_{k=-M}^{0} T_{k} \alpha_{k} \cos \left(\omega_{k}\left(t-\frac{\Delta \tau}{2}\right)+\varphi_{k}\right) \\
\sin \phi \sqrt{\gamma} \sum_{k=-M}^{0} T_{k} \alpha_{k} \cos \left(\omega_{k}\left(t+\frac{\Delta \tau}{2}\right)+\varphi_{k}\right)
\end{array}\right)+\left(\begin{array}{l}
\frac{1}{\sqrt{2}} \sum_{k=-M}^{0} T_{k} n_{k} \cos \left(\omega_{k} t+\varphi_{x r k}\right) \\
\frac{1}{\sqrt{2}} \sum_{k=-M}^{0} T_{k} n_{k} \cos \left(\omega_{k} t+\varphi_{y r k}\right)
\end{array}\right)
\end{gathered}
$$




$$
\overrightarrow{E_{U S B}}(t)=\left(\begin{array}{l}
\cos \phi \sum_{k=0}^{M} T_{k} \alpha_{k} \cos \left(\omega_{k}\left(t-\frac{\Delta \tau}{2}\right)+\varphi_{k}\right) \\
\sin \phi \sqrt{\gamma} \sum_{k=0}^{M} T_{k} \alpha_{k} \cos \left(\omega_{k}\left(t+\frac{\Delta \tau}{2}\right)+\varphi_{k}\right.
\end{array}\right)+\left(\begin{array}{l}
\frac{1}{\sqrt{2}} \sum_{k=0}^{M} T_{k} n_{k} \cos \left(\omega_{k} t+\varphi_{x r k}\right) \\
\frac{1}{\sqrt{2}} \sum_{k=0}^{M} T_{k} n_{k} \cos \left(\omega_{k} t+\varphi_{y r k}\right)
\end{array}\right)
$$

The fields at the output of the two PBS are referred to as $\left(E_{U F}\right.$, $\left.E_{U S}\right)$ for the fast and slow polarizations of the USB, and $\left(E_{L F}\right.$, $E_{L S}$ ) for the fast and slow polarizations of the LSB:

$$
\begin{aligned}
& \left(\begin{array}{l}
\mathrm{E}_{\mathrm{UF}} \\
\mathrm{E}_{\mathrm{US}}
\end{array}\right)=\sqrt{G_{U}(t)}\left(\begin{array}{cc}
\cos \theta_{U} & \sin \theta_{U} \\
-\sin \theta_{U} & \cos \theta_{U}
\end{array}\right) \overrightarrow{E_{U S B}}(t) \\
& \left(\begin{array}{l}
\mathrm{E}_{\mathrm{LF}} \\
\mathrm{E}_{\mathrm{LS}}
\end{array}\right)=\sqrt{G_{L}(t)}\left(\begin{array}{cc}
\cos \theta_{L} & \sin \theta_{L} \\
-\sin \theta_{L} & \cos \theta_{L}
\end{array}\right) \overrightarrow{E_{L S B}}(t)
\end{aligned}
$$

where $\theta_{U}$ and $\theta_{L}$ represent, respectively, the orientation angles of the PBS on the upper and lower sidebands with respect to the PSP of the signal; $G_{U}(t)$ and $G_{L}(t)$ represent, the EAM transfer function on the upper and lower sidebands. Here we assume that the transfer functions of the two EAMs are identical and they are driven by the same local-oscillator (LO) such that $G_{U}=G_{L}=G(t)$. If we assume that the EAM is operated in the linear part of its transfer function and driven by a sinusoidal voltage, then the transfer function can be approximated by a raised cosine.

$$
G(t)=\frac{1}{2}\left(1+\cos \left(\omega_{L O} t+\varphi_{L O}\right)\right)
$$

where $\omega_{L O}$ is the modulation frequency of the optical modulator and $\theta_{L O}$ is $L O$ phase relative to the data clock. The four signals at the output of the two PBS are detected using low-speed detectors. Their intensities are referenced as $\left(I_{U F}\right.$, $\left.I_{U S}\right)$ for the fast and slow polarizations of the USB, and $\left(I_{L F}\right.$, $\left.I_{L S}\right)$ for the fast and slow polarizations of the LSB:

$$
\begin{aligned}
& \left\{\begin{array}{l}
I_{U F}(t)=G(t)\left(\cos \theta_{U} E_{U x}+\sin \theta_{U} E_{U y}\right)^{2} \\
I_{U S}(t)=G(t)\left(-\sin \theta_{U} E_{U x}+\cos \theta_{U} E_{U y}\right)^{2}
\end{array}\right. \\
& \left\{\begin{array}{l}
I_{L F}(t)=G(t)\left(\cos \theta_{L} E_{L x}+\sin \theta_{L} E_{L y}\right)^{2} \\
I_{L S}(t)=G(t)\left(-\sin \theta_{L} E_{L x}+\cos \theta_{L} E_{L y}\right)^{2}
\end{array}\right.
\end{aligned}
$$

where $\left(E_{U x}, E_{U y}\right)$ and $\left(E_{L x}, E_{L y}\right)$ are, respectively, the two polarization components on the $\mathrm{x}-\mathrm{y}$ axis. The SSB tones are down-converted to an intermediate frequency $\omega_{I F}=\omega_{T}-\omega_{L O}$ where $\omega_{T}=k_{T} \Delta \omega$ is defined such that the first tone has an index $k$ and the second tone has an index $I$ such that $I=k+k_{T}$. Expanding this and considering the filtering function of the low speed detectors will result in only IF tones of an index $l=k+k_{T}$ remaining and with a null for $l \neq k+k_{T}$. We also assume that terms containing $<\cos (\omega t)>=0$ and $<\cos \varphi_{r}>=<\sin \varphi_{r}>=0$ when $\varphi_{r}$ is random, time-average to zero to obtain (11) and (12), where $\omega_{k}=\omega_{0}+k \Delta \omega$ and $\omega_{0}, \Delta \omega$ are respectively the central optical angular-frequency of the data signal and the frequency spacing between the data tones which depends on the length of the repeating PRBS data signal that is used. If real data were used, periodic spacing would not be observed, with the spectrum becoming continuous in the limit of truly random data. As the technique relies on a comparison, the exact nature of the spectral components is not important, only that components exist within the bandwidth observed, although the use of PRBS makes the practical implementation simpler. It can be seen that the phase and amplitude of the IF tones on the slow and fast PSPs will depend on the PBS orientation angles $\left(\theta_{U}\right)$. In order to suppress this dependency, $\theta_{U}$ must be adjusted to zero, which can be achieved by tuning the polarization controller (PC) placed before each PBS. The RF power of each IF tone is used as a control feed-back to tune the PCs. Within one sideband, the maximum RF power of the fast polarization IF tone (corresponding to the minimum RF power of the slow polarization IF tone) coincide with the alignment of the PBS and signal's PSPs $\left(\theta_{U}=0\right)$.

$$
\theta_{U}=n \pi:\left\{\begin{array}{l}
\left|I_{U F}\left(\omega_{I F}\right)\right|^{2}=\operatorname{Max}\left|I_{U F}\left(\omega_{I F}\right)\right|^{2} \\
\left|I_{U S}\left(\omega_{I F}\right)\right|^{2}=\operatorname{Min}\left|I_{U S}\left(\omega_{I F}\right)\right|^{2}
\end{array}\right.
$$

In this configuration where $\theta_{U}=0$, the full expression of the USB IF tones is derived from (11) and (12) is given by:

$$
\begin{aligned}
& \text { for } l=k+k_{T} \\
& I_{U F}\left(\omega_{I F}\right)=\frac{1}{16}\left(\begin{array}{l}
\cos ^{2} \phi \cos ^{2} \theta_{U} \exp i\left(\varphi_{U S B}-\varphi_{L O}-\frac{k_{T} \Delta \omega \Delta \tau}{2}\right) \sum_{k=0}^{M} \sum_{l=k+k_{T}}^{M} T_{k} T_{l} \alpha_{k} \alpha_{l} \\
+\sin ^{2} \phi \sin ^{2} \theta_{U} \exp i\left(\varphi_{U S B}-\varphi_{L O}+\frac{k_{T} \Delta \omega \Delta \tau}{2}\right) \sum_{k=0}^{M} \sum_{l=k+k_{T}}^{M} T_{k} T_{l} \alpha_{k} \alpha_{l} \\
\left.+2 \sin \phi \cos \phi \sin \theta_{U} \cos \theta_{U} \exp i\left(\varphi_{U S B}-\varphi_{L O}-\left(\omega_{k+\Delta k}+\omega_{k}\right) \frac{\Delta \tau}{2}\right) \sum_{k=0}^{M} \sum_{l=k+k_{T}}^{M} T_{k} T_{l} \alpha_{k} \alpha_{l}\right) \\
\text { for } l \neq k+k_{T} \\
I_{U F}\left(\omega_{I F}\right)=0
\end{array}\right.
\end{aligned}
$$




$$
\begin{aligned}
& \text { for } l=k+k_{T} \\
& I_{U S}\left(\omega_{I F}\right)=\frac{1}{16}\left(\begin{array}{l}
\cos ^{2} \phi \sin ^{2} \theta_{U} \exp i\left(\varphi_{U S B}-\varphi_{L O}-\frac{k_{T} \Delta \omega \Delta \tau}{2}\right) \sum_{k=0}^{M} \sum_{l=k+k_{T}}^{M} T_{k} T_{l} \alpha_{k} \alpha_{l} \\
+\sin ^{2} \phi \cos ^{2} \theta_{U} \exp i\left(\varphi_{U S B}-\varphi_{L O}+\frac{k_{T} \Delta \omega \Delta \tau}{2}\right) \sum_{k=0}^{M} \sum_{l=k+k_{T}}^{M} T_{k} T_{l} \alpha_{k} \alpha_{l} \\
-2 \sin \phi \cos \phi \sin \theta_{U} \cos \theta_{U} \exp i\left(\varphi_{U S B}-\varphi_{L O}-\left(\omega_{k+\Delta k}+\omega_{k}\right) \frac{\Delta \tau}{2}\right) \sum_{k=0}^{M} \sum_{l=k+k_{T}}^{M} T_{k} T_{l} \alpha_{k} \alpha_{l}
\end{array}\right)
\end{aligned}
$$

$$
\text { for } l \neq k+k_{T} \quad I_{U S}\left(\omega_{I F}\right)=0
$$

for $l=k+k_{T}$ :

$$
\begin{aligned}
& I_{U F}\left(\omega_{I F}\right)=\frac{\cos ^{2} \phi}{16} \mathrm{e}^{i\left(\varphi_{U S B}-\varphi_{L O}-\frac{k_{T} \Delta \omega \Delta \tau}{2}\right)} \sum_{k=0}^{M} \sum_{l=k+k_{T}}^{M} T_{k} T_{l} \alpha_{k} \alpha_{l} \\
& I_{U S}\left(\omega_{I F}\right)=\frac{\sin ^{2} \phi}{16} \mathrm{e}^{i\left(\varphi_{U S B}-\varphi_{L O}+\frac{k_{T} \Delta \omega \Delta \tau}{2}\right)} \sum_{k=0}^{M} \sum_{l=k+k_{T}}^{M} T_{k} T_{l} \alpha_{k} \alpha_{l} \\
& \text { for } l \neq k+k_{T}: \quad I_{U F}\left(\omega_{I F}\right)=0 \quad I_{U S}\left(\omega_{I F}\right)=0
\end{aligned}
$$

Similar analysis shows that for $\theta_{L}=0$, the full expression of the LSB IF tones $I_{L F}\left(\omega_{I F}\right)$ and $I_{L S}\left(\omega_{I F}\right)$ are given by:

for $l=k+k_{T}$ :

$$
\begin{aligned}
& I_{L F}\left(\omega_{I F}\right)=\frac{\cos ^{2} \phi}{16} \mathrm{e}^{i\left(\varphi_{L S B}-\varphi_{L O}-\frac{k_{T} \Delta \omega \Delta \tau}{2}\right)} \sum_{k=-M}^{0} \sum_{l=k-k_{T}}^{0} T_{k} T_{l} \alpha_{k} \alpha_{l} \\
& I_{L S}\left(\omega_{I F}\right)=\frac{\sin ^{2} \phi}{16} \mathrm{e}^{i\left(\varphi_{L S B}-\varphi_{L O}+\frac{k_{T} \Delta \omega \Delta \tau}{2}\right)} \sum_{k=-M}^{0} \sum_{l=k-k_{T}}^{0} T_{k} T_{l} \alpha_{k} \alpha_{l} \\
& \text { for } l \neq k+k_{T}: \quad I_{L F}\left(\omega_{I F}\right)=0 \\
& I_{L S}\left(\omega_{I F}\right)=0
\end{aligned}
$$

In the following sections we show how the amplitude and phases of the four IF tones (the fast and slow polarizations of the USB and LSB) that are extracted from (14) and (15) can be used to measure OSNR, GVD and DGD.

$$
\left(\begin{array}{cc}
\varphi_{U F} & \varphi_{U S} \\
\varphi_{L F} & \varphi_{L S}
\end{array}\right)=\left(\begin{array}{ll}
\varphi_{U S B}-\varphi_{L O}-\frac{k_{T} \Delta \omega \Delta \tau}{2} & \varphi_{U S B}-\varphi_{L O}+\frac{k_{T} \Delta \omega \Delta \tau}{2} \\
\varphi_{L S B}-\varphi_{L O}-\frac{k_{T} \Delta \omega \Delta \tau}{2} & \varphi_{L S B}-\varphi_{L O}+\frac{k_{T} \Delta \omega \Delta \tau}{2}
\end{array}\right)
$$

\section{B. Analytical model of CD monitoring}

From (16), the phase difference between the IF tones from the upper and lower sidebands for each polarization state is defined by:

$$
\begin{aligned}
\Delta \varphi_{G V D_{-} F} & =\varphi_{\mathrm{UF}}-\varphi_{\mathrm{LF}}=\varphi_{U S B}-\varphi_{L S B} \\
\Delta \varphi_{G V D_{-} S} & =\varphi_{\mathrm{US}}-\varphi_{\mathrm{LS}}=\varphi_{U S B}-\varphi_{L S B}
\end{aligned}
$$

It is clearly shown that both phase differences $\Delta \varphi_{G V D} F$ and $\Delta \varphi_{G V D_{-} L}$ measured respectively, on the fast and slow polarization states, are equal to $\Delta \varphi_{G V D_{-} F}=\Delta \varphi_{G V D_{-} S}=\Delta \varphi_{G V D}$. The CD induces a time delay between the upper and lower sidebands of the optical signal, this time delay is converted into a phase shift $\Delta \varphi_{G V D}$. After transmission, the accumulated dispersion is given by:

$$
G V D=\frac{1}{2 \pi} \frac{c}{\lambda_{T}^{2}} \frac{1}{\omega_{T}^{2}} \Delta \varphi_{G V D}
$$

where $c$ is the light velocity, $\lambda_{T}$ and $\omega_{T}$ are, respectively, the optical wavelength and angular frequency of the monitored tone. It is shown analytically in (19) that $\Delta \varphi_{G V D}$ is proportional to GVD and is independent of DGD. This is the case since the DGD will introduce the same amount of phase shift to both $\left(\varphi_{U F}, \varphi_{L F}\right)$ and $\left(\varphi_{U S}, \varphi_{L S}\right)$ since PMD is, to the first order, wavelength independent. It is also noted that $\Delta \varphi_{G V D}$ is independent of noise and therefore independent of the OSNR. This is the case since the IF tones are noise independent, as shown in (14) and (15). The maximum measurement range $G V D_{\max }$ is defined such that $\Delta \varphi_{G V D}=2 \pi$ :

$$
G V D_{\max }=\frac{c}{\lambda_{T}^{2}} \frac{1}{\omega_{T}^{2}}
$$

For an optical signal of $1550 \mathrm{~nm}$ and a $5 \mathrm{GHz}$ monitored tone, the maximum monitoring range is equal to $4995 \mathrm{ps} / \mathrm{nm}$. A larger range can be obtained by reducing the monitored tone frequency at the expense of degrading the measurement accuracy. Similarly, a smaller range can be obtained by increasing the monitored tone frequency which would need the use of wider bandwidth electronics but would achieve better measurement accuracy.

\section{Analytical model of PMD monitoring}

From (17), the phase difference between the slow and fast polarization-components of the IF tones from each sideband is

$$
\begin{aligned}
& \Delta \varphi_{D G D_{-} U}=\varphi_{U S}-\varphi_{U F}=k_{T} \Delta \omega \Delta \tau=\omega_{T} \Delta \tau \\
& \Delta \varphi_{D G D_{-} L}=\varphi_{L S}-\varphi_{L F}=k_{T} \Delta \omega \Delta \tau=\omega_{T} \Delta \tau
\end{aligned}
$$

It is shown analytically in (21) and (22) that $\Delta \varphi_{D G D_{-} U}$ and $\triangle \varphi_{D G D_{-} L}$ are proportional to DGD and are independent of GVD. Since both sidebands are monitored at the same frequency, it is also shown that both phase differences, $\Delta \varphi_{D G D_{-} U}$ and $\Delta \varphi_{D G D_{-} L}$ are equal, thus it is sufficient to conduct measurements on one sideband to extract DGD:

$$
\Delta \varphi_{D G D_{-} U}=\Delta \varphi_{D G D_{-} L}=\Delta \varphi_{D G D}=\omega_{T} \Delta \tau
$$

Similarly to the CD measurement, we see that $\Delta \varphi_{D G D}$ is independent of noise and therefore independent of the OSNR. This can be confirmed by noting that the IF tones are noise independent. The maximum measurement range $\Delta \tau_{\max }$ is 
for $l=k \quad I_{\mathrm{UF}}(t)=\frac{1}{4}\left(\begin{array}{l}\cos ^{2} \phi \sum_{k=0}^{M} T_{k}^{2} \alpha_{k}^{2}+\frac{1}{2} \sum_{k=0}^{M} T_{k}^{2} n_{k}^{2} \\ +\frac{2}{\sqrt{2}} \cos \phi \sum_{k=0}^{M} T_{k}^{2} \alpha_{k} n_{k} \cos \left(\omega_{k} \frac{\Delta \tau}{2}+\left(\varphi_{x x k}-\varphi_{k}\right)\right)\end{array}\right)++\frac{1}{8}\left(\begin{array}{l}\cos ^{2} \phi \sum_{k=0}^{M} T_{k}^{2} \alpha_{k}^{2} \cos \left(\omega_{L O} t+\varphi_{L O}\right)+\frac{1}{2} \sum_{k=0}^{M} T_{k}^{2} n_{k}^{2} \cos \left(\omega_{L O} t+\varphi_{L O}\right) \\ +\frac{2}{\sqrt{2}} \cos \phi \sum_{k=0}^{M} T_{k}^{2} \alpha_{k} n_{k} \cos \left(\omega_{I F} t+\omega_{k} \frac{\Delta \tau}{2}+\varphi_{L O}-\left(\varphi_{x x k}-\varphi_{k}\right)\right)\end{array}\right)$

for $l \neq k \quad I_{\mathrm{UF}}(t)=0$

for $l=k \quad I_{\mathrm{US}}(t)=\frac{1}{4}\left(\begin{array}{l}\sin ^{2} \phi \sum_{k=0}^{M} T_{k}^{2} \alpha_{k}^{2}+\frac{1}{2} \sum_{k=0}^{M} T_{k}^{2} n_{k}^{2} \\ +\frac{2}{\sqrt{2}} \sin \phi \sum_{k=0}^{M} T_{k}^{2} \alpha_{k} n_{k} \cos \left(-\omega_{k} \frac{\Delta \tau}{2}+\left(\varphi_{y r k}-\varphi_{k}\right)\right.\end{array}\right)++\frac{1}{8}\left(\begin{array}{l}\sin ^{2} \phi \sum_{k=0}^{M} T_{k}^{2} \alpha_{k}^{2} \cos \left(\omega_{L O} t+\varphi_{L O}\right)+\frac{1}{2} \sum_{k=0}^{M} T_{k}^{2} n_{k}^{2} \cos \left(\omega_{I F} t+\varphi_{L O}\right) \\ +\frac{2}{\sqrt{2}} \sin \phi \sum_{k=0}^{M} T_{k}^{2} \alpha_{k} n_{k} \cos \left(\omega_{L O} t-\omega_{k} \frac{\Delta \tau}{2}+\varphi_{L O}-\left(\varphi_{y r k}-\varphi_{k}\right)\right)\end{array}\right)$

for $l=k \quad I_{\mathrm{US}}(t)=0$

defined such that $\Delta \varphi_{D G D}=2 \pi$ and $\Delta \tau_{\max }=2 \pi / \omega_{T}$. For a $5 \mathrm{GHz}$ monitored tone, the maximum monitoring range is equal to 200 ps. Similarly to the CD range analysis mentioned above, a larger DGD range can be obtained by reducing the monitored tone frequency at the expense of degrading the measurement accuracy.

\section{Analytical model of OSNR monitoring}

Here we show that the signal average-power of a sideband in conjunction with the IF tone amplitude measurement of the same sideband provides a measure of OSNR. The signal's average power is the result of the beating terms between any two tones of equal optical frequency. In this case, the expression of the signals average power is obtained from $I_{U F}(t)$ for an index $l=k$ and null for $l \neq k$. This can be demonstrated as (24) and (25) for $\theta_{U}=\theta_{L}=0$ and $l=k$.

Since $<\cos (\omega t)>=0$ and $<\cos \varphi_{r}>=<\sin \varphi_{r}>=0$, it is shown that the time-averaged detected signals $\left\langle I_{U F}(t)>\right.$ and $\left\langle I_{U S}(t)>\right.$ are linearly proportional to the sum of the USB signal averagepower $\sum_{k=0}^{M} T_{k}^{2} \alpha_{k}^{2}$ and the noise power $\sum_{k=0}^{M} T_{k}^{2} n_{k}^{2}$ :

$$
\left\{\begin{array}{l}
<I_{U F}(t)>=\frac{1}{4}\left(\cos ^{2} \phi \sum_{k=0}^{M} T_{k}^{2} \alpha_{k}^{2}+\frac{1}{2} \sum_{k=0}^{M} T_{k}^{2} n_{k}^{2}\right) \\
<I_{U S}(t)>=\frac{1}{4}\left(\sin ^{2} \phi \sum_{k=0}^{M} T_{k}^{2} \alpha_{k}^{2}+\frac{1}{2} \sum_{k=0}^{M} T_{k}^{2} n_{k}^{2}\right)
\end{array}\right.
$$

However, both signals $\left\langle I_{U F}(t)>\right.$ and $\left\langle I_{U S}(t)>\right.$ are dependent on $\phi$, the angle between the linearly polarized signal and the fast principal state, which would make the OSNR measurement dependent on the incoming signal polarization state. In order to suppress this dependency, the sum of both signals $\left\langle I_{U F}(t)>\right.$ and $\left\langle I_{U S}(t)>\right.$ is calculated to be:

$$
<I_{U}(t)>=<I_{U F}(t)>+<I_{U S}(t)>=\frac{1}{4} \sum_{k=0}^{M} T_{k}^{2}\left(\alpha_{k}^{2}+n_{k}^{2}\right)
$$

The signal $<I_{U}(t)>$ becomes solely dependent on the incoming signal average-power and noise power, and independent of PMD and CD. We define $I_{0}$ as the time-averaged signal power in the absence of noise:

$$
I_{0}=<I_{U}(t)>\left.\right|_{n=0}=\frac{1}{4} \sum_{k=0}^{1} T_{k}^{2} \alpha_{k}^{2}
$$

and the total noise power $n^{2}$ is defined as:

$$
n^{2}=\frac{1}{4} \sum_{k=0}^{1} T_{k}^{2} n_{k}^{2}
$$

Such that the time-averaged detected signal $\left\langle I_{U}(t)\right\rangle$ can be expressed as (see $(27)<I_{U}(t)>=I_{0}+n^{2}$. It is shown in (14) that the amplitude of the IF tones $\left|I_{U F}\left(\omega_{\mathrm{IF}}\right)\right|$ and $\left|I_{U S}\left(\omega_{\mathrm{IF}}\right)\right|$, depends on the quantity $\sum_{k=0}^{M} \sum_{l=k+k_{T}}^{M} T_{k} T_{l} \alpha_{k} \alpha_{l}$ which is independent of the noise power:

$$
\left\{\begin{array}{l}
\left|I_{U F}\left(\omega_{I F}\right)\right|=\frac{\cos ^{2} \phi}{16} \sum_{k=0}^{M} \sum_{l=k+k_{T}}^{M} T_{k} T_{l} \alpha_{k} \alpha_{l} \\
\left|I_{U S}\left(\omega_{I F}\right)\right|=\frac{\sin ^{2} \phi}{16} \sum_{k=0}^{M} \sum_{l=k+k_{T}}^{M} T_{k} T_{l} \alpha_{k} \alpha_{l}
\end{array}\right.
$$

However, both quantities $\left|I_{U F}\left(\omega_{\mathrm{IF}}\right)\right|$ and $\left|I_{U S}\left(\omega_{\mathrm{IF}}\right)\right|$ are dependent on $\phi$, which would also make the OSNR measurement dependent on the incoming signal polarization state. In order to suppress this dependency, the sum of both quantities $\left|I_{U F}\left(\omega_{\mathrm{IF}}\right)\right|$ and $\left|I_{U S}\left(\omega_{\mathrm{IF}}\right)\right|$ is calculated to be:

$$
\left|I_{U}\left(\omega_{I F}\right)\right|=\left|I_{U F}\left(\omega_{I F}\right)\right|+\left|I_{U S}\left(\omega_{I F}\right)\right|=\sum_{k=0}^{M} \sum_{l=k+k_{T}}^{M} T_{k} T_{l} \alpha_{k} \alpha_{l}
$$

The quantity $\left|I_{U}\left(\omega_{I F}\right)\right|$ is proportional to the sum of all beating terms between any two tones separated by the monitored tone frequency (i.e. for $l=k+k_{T}$ ) and is independent of the noise power, the PMD and CD. It can easily be seen that the IF tone amplitude defined in (31)is linearly proportional to the signal power $I_{0}$. This can be demonstrated as follows: Let us consider that $I_{0}$ is amplified by a gain factor $G$. In this case, both $I_{0}$ and $\left|I_{U}\left(\omega_{I F}\right)\right|$ can be written as follows

$$
\left\{\begin{array}{l}
\frac{1}{4} \sum_{k=0}^{1}\left(\sqrt{G} T_{k} \alpha_{k}\right)^{2}=G \frac{1}{4} \sum_{k=0}^{1} T_{k}^{2} \alpha_{k}^{2}=G I_{0} \\
\sum_{k=0}^{M} \sum_{l=k+k_{T}}^{M}\left(\sqrt{G} T_{k} \alpha_{k}\right)\left(\sqrt{G} T_{l} \alpha_{l}\right)=G \sum_{k=0}^{M} \sum_{l=k+k_{T}}^{M} T_{k} T_{l} \alpha_{k} \alpha_{l}=G\left|I_{U}\left(\omega_{l F}\right)\right|
\end{array}\right.
$$

Thus the IF tone amplitude can be written as a function of $I_{0}$ as

$$
\left|I_{U}\left(\omega_{I F}\right)\right|=\rho^{2} I_{0}^{2}
$$

where $\rho$ is a constant of proportionality. The ratio $\left(R_{U}\right)$ of the USB signal average-power (signal and noise) from (27) to the USB IF tone amplitude (signal only) (31) is inversely proportional to the signal-to-noise ratio, and is defined as: 


$$
R_{U}(M)=\frac{<I_{U}(t)>}{\left|I_{U}\left(\omega_{I F}\right)\right|}=\frac{1}{4} \frac{\sum_{k=0}^{M} T_{k}^{2}\left(\alpha_{k}^{2}+n_{k}^{2}\right)}{\sum_{k=0}^{M} \sum_{l=k+k_{T}}^{M} T_{k} T_{l} \alpha_{k} \alpha_{l}}
$$

Similar analysis can be done for the LSB. The ratio RL can be derived from the previous by considering tones with negative indexes:

$$
R_{L}(M)=\frac{<I_{L}(t)>}{\left|I_{L}\left(\omega_{I F}\right)\right|}=\frac{1}{4} \frac{\sum_{k=-M}^{0} T_{k}^{2}\left(\alpha_{k}^{2}+n_{k}^{2}\right)}{\sum_{k=-M}^{0} \sum_{l=k-k_{T}}^{0} T_{k} T_{l} \alpha_{k} \alpha_{l}}
$$

It is assumed that the power spectrum of the LSB and USB are symmetrical around the carrier which is justified by the use of a MZI filter with a symmetrical transfer function. In this case, the sum from $-M$ to 0 in (34) can be replaced by the sum from 0 to $M$ leading to the ratio $R_{L}$ being equal to $R_{U}$ which will shows that one sideband measurement is sufficient for the OSNR monitoring as $R_{U}(M)=R_{L}(M)=R(M)$. In order to illustrate the relationship of $R$ to OSNR, the total signal power, $R$ can be expressed as:

$$
R=\frac{I_{0}+n^{2}}{\rho I_{0}}=\frac{1}{\rho}\left(1+\frac{n^{2}}{I_{0}}\right)
$$

This ratio $R$ is related to the OSNR as follows

$$
O S N R=-10 \log _{10}\left(\eta \frac{n^{2}}{I_{0}}\right)=-10 \log _{10}(\eta \rho R-\eta)
$$

where $\eta$ is a constant of proportionality, dependent on the effective measurement bandwidth. The OSNR can then be determined from the measurement of $R$ obtained from the ratio of the total power to the IF tone amplitude.

\section{TOLERANCE TO OPTICAL FILTER IMPERFECTIONS}

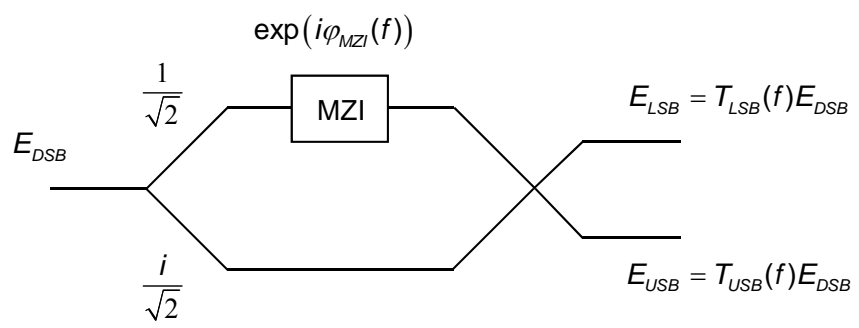

Fig5: MZI filter device structure.

One of the most important elements in this scheme is the MZI filter which is used to filter each sideband from the dualsideband incoming signal. The filter is tuned and maintained at a given wavelength such that each output port passes one sideband and suppresses the other sideband. It is under this configuration, corresponding to zero detuning, where the setup calibration is performed for all impairment monitoring. Two possible defects may affect the performance of the system. If the filter drifts from its desired position of zero detuning, the amplitude and phase of the monitored tones can be altered due to the amplitude and phase response of the filter. Secondly, as demonstrated in Figure 4 the filter will not entirely reject the unwanted sideband. Figure 5 shows the diagram of the filter, based on a Mach-Zehnder interferometer structure. The phase-shift $\varphi_{\text {MZI }}(f)$ introduced in one arm of the interferometer is given by:

$$
\varphi_{\mathrm{MZI}}(f)=2 \pi\left(\frac{f-f_{T}}{f_{F S R}}\right)
$$

Where $f_{T}$ is the monitored tone frequency and $f_{F S R}$ is the filter's FSR. In order to transmit one sideband and suppress the other, an inherent relationship between $f_{T}$ and $f_{F S R}$ is such that $f_{F S R}=4 f_{T}$ (see Figure 6). In a WDM system, the MZI FSR is chosen as a sub-multiple of the channel spacing in order to transmit the USB and suppress the LSB of all channels simultaneously. In the experimental work described in the next chapter, $f_{T}$ was equal to $\sim 5 \mathrm{GHz}, f_{F S R}$ equal to $20 \mathrm{GHz}$ with a WDM channel spacing of $100 \mathrm{GHz}$. The MZI amplitude transfer-function for both the lower and upper sidebands can be modeled as follows:

$$
\left\{\begin{array}{l}
T_{L S B}(f)=i \cos \left(\frac{1}{2} \varphi_{\mathrm{MZI}}(f)\right) \exp \left(\frac{i}{2} \varphi_{\mathrm{MZI}}(f)\right) \\
T_{U S B}(f)=i \sin \left(\frac{1}{2} \varphi_{\mathrm{MZI}}(f)\right) \exp \left(\frac{i}{2} \varphi_{\mathrm{MZI}}(f)\right)
\end{array}\right.
$$

Figure 6 shows the diagram of the filter's detuning direction in relation to the data signal. In order to illustrate the effect of the filter's detuning on the proposed monitoring technique, the filtered SSB optical field can be modeled as two monochromatic tones where $M=1$ and $K_{T}=1$, referred to as a two-tone model thus greatly simplifying the filter detuning analytical results. Using the two-tone model, the expression of the IF tones, is then given by (14).

$$
\left\{\begin{array}{l}
I_{U F}\left(\omega_{I F}\right)=\frac{\cos ^{2} \phi}{16} \exp i\left(\varphi_{U S B}-\varphi_{L O}-\frac{k_{T} \Delta \omega \Delta \tau}{2}\right) T_{0} T_{1} \alpha_{0} \alpha_{1} \\
I_{U S}\left(\omega_{I F}\right)=\frac{\sin ^{2} \phi}{16} \exp i\left(\varphi_{U S B}-\varphi_{L O}+\frac{k_{T} \Delta \omega \Delta \tau}{2}\right) T_{0} T_{1} \alpha_{0} \alpha_{1}
\end{array}\right.
$$

where $T_{0}=T(0)$ and $T_{1}=T\left(f_{T}\right)$, the filter's transfer-function, respectively, at the carrier and the monitored tone frequency.

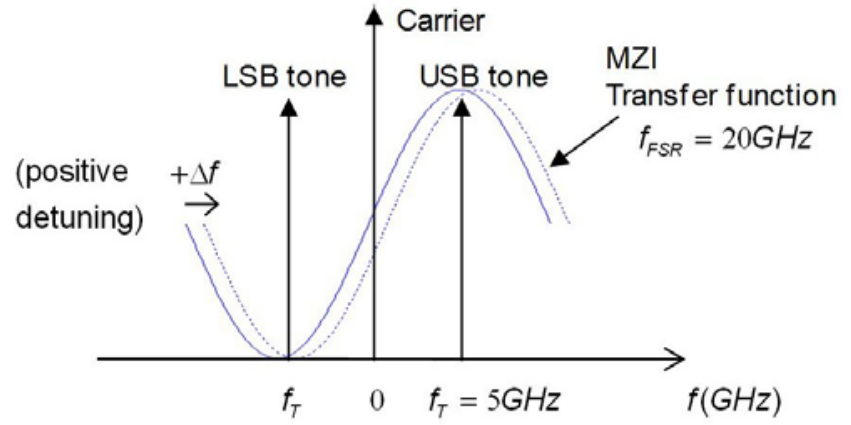

Fig 6: MZI filter detuning.

In order to assess the filter's detuning effect on PMD and CD monitoring, the phase of the four monitored tones is reassessed taking into account the filter's phase contribution. The phases of the two USB IF tones are: 


$$
\left\{\begin{array}{l}
\varphi_{U F}=\varphi_{U S B}-\varphi_{L O}-\frac{k_{T} \Delta \omega \Delta \tau}{2}+\frac{1}{2} \varphi_{\mathrm{MZI}}(0)+\frac{1}{2} \varphi_{\mathrm{MZI}}\left(f_{T}\right)+\frac{\pi}{2} \\
\varphi_{U S}=\varphi_{U S B}-\varphi_{L O}+\frac{k_{T} \Delta \omega \Delta \tau}{2}+\frac{1}{2} \varphi_{\mathrm{MZI}}(0)+\frac{1}{2} \varphi_{\mathrm{MZI}}\left(f_{T}\right)+\frac{\pi}{2}
\end{array}\right.
$$

And the phases of the two LSB IF tones are:

$$
\left\{\begin{array}{l}
\varphi_{L F}=\varphi_{L S B}-\varphi_{L O}-\frac{k_{T} \Delta \omega \Delta \tau}{2}+\frac{1}{2} \varphi_{\mathrm{MZI}}(0)+\frac{1}{2} \varphi_{\mathrm{MZI}}\left(f_{T}\right)+\frac{\pi}{2} \\
\varphi_{L S}=\varphi_{L S B}-\varphi_{L O}+\frac{k_{T} \Delta \omega \Delta \tau}{2}+\frac{1}{2} \varphi_{\mathrm{MZI}}(0)+\frac{1}{2} \varphi_{\mathrm{MZI}}\left(f_{T}\right)+\frac{\pi}{2}
\end{array}\right.
$$

From the equations above, it is shown that (23) remains valid, where $\Delta \varphi_{D G D}=k_{T} \Delta \omega \Delta \tau$ and $\Delta \varphi_{D G D=} \varphi_{U S B}-\varphi_{L S B}$ are both independent of the filter's phase impact. This is the case since the MZI filter introduces the same phase shift for each monitored tone. The MZI filter's detuning effect on OSNR is also assessed. With no detuning, the ratio $R$ for the USB can be derived from (34) when considering a two-tone model $(M=1)$ :

$$
R(\Delta f=0)=\frac{<I_{U}(t)>}{\left|I_{U}\left(\omega_{I F}\right)\right|}=\frac{1}{4} \frac{|T(0)|^{2}\left(\alpha_{0}^{2}+n_{0}^{2}\right)+\left|T\left(f_{T}\right)\right|^{2}\left(\alpha_{1}^{2}+n_{1}^{2}\right)}{|T(0)|\left|T\left(f_{T}\right)\right| \alpha_{0} \alpha_{1}}
$$

where $T_{0}=T(f=0)$ and $T_{l}=T\left(f=f_{T}\right)$. By introducing a filter detuning $\Delta f$, the expression of $\mathrm{R}$ becomes:

$$
R(\Delta f)=\frac{<I_{U}(t)>}{\left|I_{U}\left(\omega_{I F}\right)\right|}=\frac{1}{4} \frac{|T(\Delta f)|^{2}\left(\alpha_{0}^{2}+n_{0}^{2}\right)+\left|T\left(f_{T}+\Delta f\right)\right|^{2}\left(\alpha_{1}^{2}+n_{1}^{2}\right)}{|T(\Delta f)| T\left(f_{T}+\Delta f\right) \mid \alpha_{0} \alpha_{1}}
$$

It is shown from (44) that the ratio R, thus the OSNR measurement, is affected by the filter's detuning, since $|T(0)| \neq$ $|T(\Delta f)|$ and $\left|T\left(f_{T}\right)\right| \neq\left|T\left(f_{T}+\Delta f\right)\right|$. This is the case since detuning the filter changes both the USB average-power and the IF tone's amplitude.

\section{EXPERIMENTAL RESUlTS}
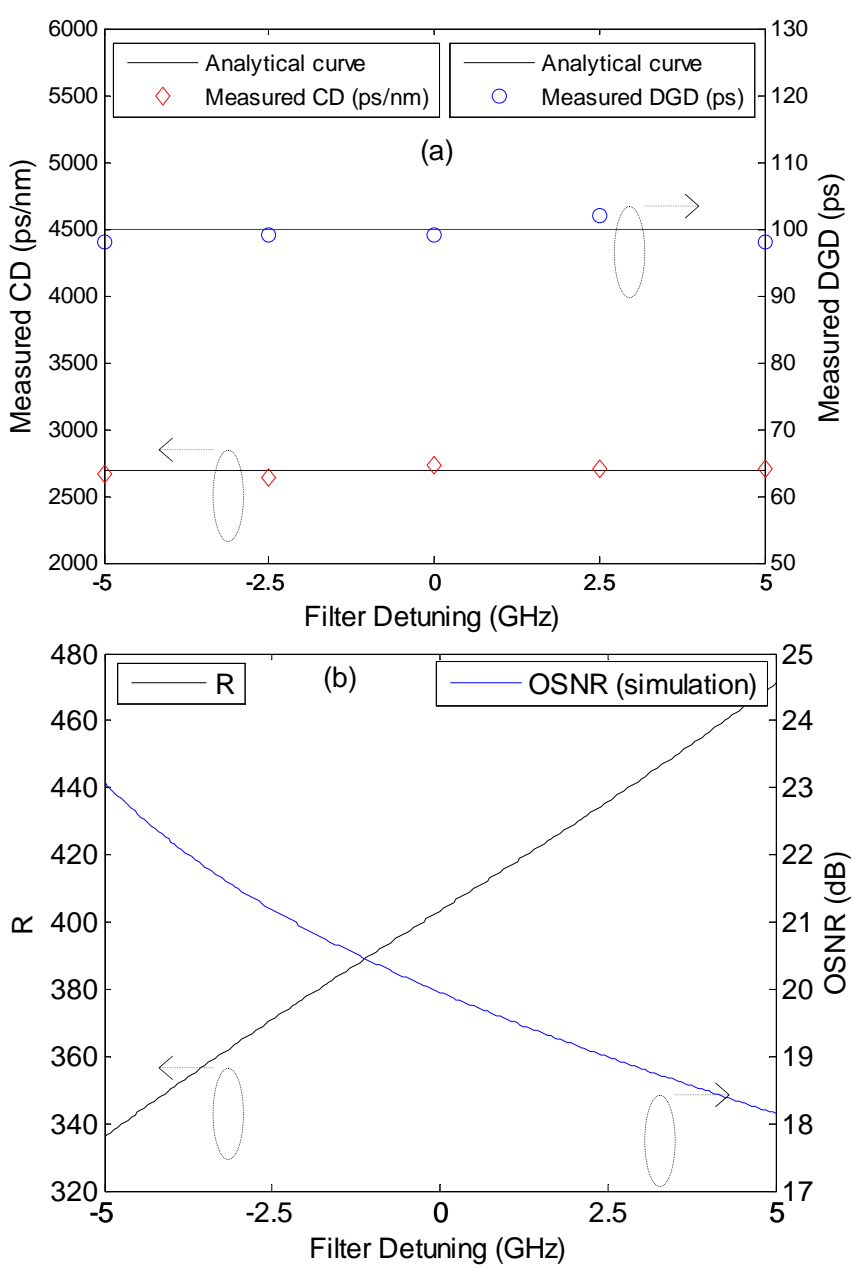

Fig 7: (a) Experimental results showing the filter detuning effect on $\mathrm{CD}$ and DGD measurements $(\mathrm{DC}=2685 \mathrm{ps} / \mathrm{nm}, \mathrm{DGD}=100 \mathrm{ps})$, (b) simulation results showing the filter detuning effect on the factor $R$ and OSNR (OSNR=20dB).

An experimental setup for the simultaneous GVD, DGD and OSNR measurement was reported in [9]. The data signal is a multi-channel $39.81312 \mathrm{Gbit} / \mathrm{s}, 2^{15}-1$ pseudorandom binary sequence (PRBS) non-return-to-zero (NRZ) with a monitored tone frequency $\left(\mathrm{f}_{T}\right)$ of $4.977250 \mathrm{GHz}$ (a sub-harmonic of the data-rate). In the experiment an IF tone of $10 \mathrm{kHz}$ is detected by $100 \mathrm{kHz}$ receivers and sampled at $300 \mathrm{kS} / \mathrm{s}$. The experimental results and the theoretical range for this tone are presented in Table 1. The impact of the other impairments on the impairment of interest are also shown, confirming that each impairment can be accurately determined independently of the other impairments.

To demonstrate the effect of filter detuning the filter has been detuned experimentally by $5 \mathrm{GHz}$, in $2.5 \mathrm{GHz}$ steps, from either side of the ITU grid position and the impact this has on the GVD and DGD results measured. For this investigation the GVD, DGD are set to $2685 \mathrm{ps} / \mathrm{nm}$ and $100 \mathrm{ps}$ respectively with a system OSNR of 20dB. Fig 7(a) shows the MZI filter detuning effect on the $\mathrm{CD}$ and DGD measurement. Both impairments remain constant within their measurement error of $\pm 100 \mathrm{ps} / \mathrm{nm}$ and $\pm 4 \mathrm{ps}$ for CD and DGD respectively. Fig 
7(b) shows simulation results of the MZI filter detuning effect on the ratio $R$ and the OSNR measurement, demonstrating that the ratio $R$ increases between 336 and 470 with a detuning of $+/-5 \mathrm{GHz}$. This corresponds to an OSNR value decreasing from 23 to $18.3 \mathrm{~dB}$ with an actual value of $20 \mathrm{~dB}$ at zero detuning.

These experiments show that phase-based measurements (thus $\mathrm{CD}$ and PMD) are insensitive to filter detuning; however, amplitude-based measurements (thus OSNR) are very sensitive to the filter detuning. In a field deployment, such a monitoring technique would have to maintain the filter detuning stability at $\pm 2 \mathrm{GHz}$ of the ITU Grid wavelength to achieve an OSNR accuracy of $\pm 1 \mathrm{~dB}$ which may limit the usability of this technique for high accuracy OSNR measurements.

Table 1: Summary of Experimental results for GVD, DGD and OSNR.

\begin{tabular}{|l|l|l|l|l|l|}
\hline Impairment & $\begin{array}{l}\text { Range } \\
(\text { Theory) }\end{array}$ & $\begin{array}{l}\text { RMS Error } \\
\text { over full } \\
\text { range }\end{array}$ & $\begin{array}{l}\text { Variation } \\
\text { with GVD } \\
\left(\begin{array}{r}4742 \mathrm{ps} / \mathrm{nm} \\
\end{array}\right.\end{array}$ & $\begin{array}{l}\text { Variation } \\
\text { with DGD } \\
(0 \quad \text { to } \\
200 \mathrm{ps})\end{array}$ & $\begin{array}{l}\text { Variation } \\
\text { with } \\
\text { OSNR (10 } \\
\text { to 30dB) }\end{array}$ \\
\hline $\mathrm{CD}$ & $\begin{array}{l}4742 \mathrm{ps} / \mathrm{nm} \\
(4992 \\
\mathrm{ps} / \mathrm{nm})\end{array}$ & $\begin{array}{l} \pm 100 \mathrm{ps} / \mathrm{nm} \\
(2 \%)\end{array}$ & $\mathrm{N} / \mathrm{A}$ & $<100 \mathrm{ps} / \mathrm{nm}$ & $<100 \mathrm{ps} / \mathrm{nm}$ \\
\hline PMD & $\begin{array}{l}203 \mathrm{ps} \\
(200 \mathrm{ps})\end{array}$ & $\pm 4 \mathrm{ps}(2 \%)$ & $<4 \mathrm{ps}$ & $\mathrm{N} / \mathrm{A}$ & $<4 \mathrm{ps}$ \\
\hline OSNR & $25 \mathrm{~dB}$ & $1 \mathrm{~dB}(4 \%)$ & $<1 \mathrm{~dB}$ & $<1 \mathrm{~dB}$ & $\mathrm{~N} / \mathrm{A}$ \\
\hline
\end{tabular}

\section{CONCLUSIONS}

This paper details demonstrates a novel technique compliant with multi-channel impairment monitoring for optical networks. It derives an analytical expression for the three impairments considered, CD, PMD and OSNR and shows that the CD, PMD and OSNR can be measured independently of each other. It concludes by showing a practical implementation and demonstrating the performance of the system. It shows that the range and accuracy from GVD, DGD and OSNR are $4742 \pm 100 \mathrm{ps} / \mathrm{nm}, 200 \pm 4 \mathrm{ps} /$ and $25 \pm 1 \mathrm{~dB}$ respectively for a $5 \mathrm{GHz}$ monitored tone.

\section{REFERENCES}

[1] D.T. Neilson, D. Stiliadis, "Ultra-high capacity IP routers for the routers of tomorrow: IRIS projects," in European Conf. Opt. Comm. (ECOC), vol. 5, paper, We 1.1.4, 2005.

[2] O. Gerstel, H. Raza, "On the synergy between the electrical and photonic switching," IEEE Communications magazine, pp. 98-104, April 2003.

[3] M. Duser, "Investigation of the impact of traffic growth and variability on future network architectures ," in European Conf. Opt. Comm. (ECOC), vol. 5, Paper Tu. 4.1.2, 2005.

[4] D. C. Kilper, R. Bach, D. J. Blumenthal, D. Einstein, T. Landolsi, L. Ostar, M. Preiss, A. E. Willner, "Optical Performance Monitoring," IEEE Journal of Lightwave Technol., vol. 22, no. 1, pp. 294-304, Jan. 2004.

[5] L. Meflah-Baker, B.C. Thomsen, J.E. Mitchell and P. Bayvel, "Simultaneous chromatic dispersion, polarisation mode-dispersion and OSNR monitoing at 40Gbit/s" Optics Express 16 (2), 2008.

[6] I.P. Kaminow, T.Li, A.E. Willner, "Optical Fiber Telecommunications V B: Systems and Networks", Academic Press, p233, 2008

[7] K-P. Ho, J. M. Kahn, "Spectrum of Externally Modulated Optical Signals," IEEE J. Lightwave Technol., vol. 22, no. 2, 2004.

[8] D. Marcuse, 'Calculation of Bit-Error Probability for a Lightwave System with Optical Amplifiers and Post-Detetection Gaussian Noise', IEEE J. Lightwave Technol., vol. 9, no. 4, pp. 505-513, 1991.
[9] C. D. Poole, R. E. Wagner, "Phenomenological Approach to Polarisation Dispersion in Long Single-Mode Fibres," IEE Electron. Lett., vol. 22 , no. 19, pp. 1029-1030, 1986 